\title{
Surface composition of ordered alloys: An off-stoichiometric effect
}

\author{
Ruban, Andrei
}

Published in:

Physical Review B Condensed Matter

Link to article, DOI:

10.1103/PhysRevB.65.174201

Publication date:

2002

Document Version

Publisher's PDF, also known as Version of record

Link back to DTU Orbit

\section{Citation $(A P A)$ :}

Ruban, A. (2002). Surface composition of ordered alloys: An off-stoichiometric effect. Physical Review $B$ Condensed Matter, 65(17), 174201. https://doi.org/10.1103/PhysRevB.65.174201

\section{General rights}

Copyright and moral rights for the publications made accessible in the public portal are retained by the authors and/or other copyright owners and it is a condition of accessing publications that users recognise and abide by the legal requirements associated with these rights.

- Users may download and print one copy of any publication from the public portal for the purpose of private study or research.

- You may not further distribute the material or use it for any profit-making activity or commercial gain

- You may freely distribute the URL identifying the publication in the public portal

If you believe that this document breaches copyright please contact us providing details, and we will remove access to the work immediately and investigate your claim 


\title{
Surface composition of ordered alloys: An off-stoichiometric effect
}

\author{
A. V. Ruban \\ Center for Atomic-scale Materials Physics and Department of Physics, Technical University of Denmark, DK-2800 Lyngby, Denmark
}

(Received 6 November 2001; published 11 April 2002)

\begin{abstract}
I predict the existence of an off-stoichiometric effect in ordered alloys in the form of a distinct transition in the surface segregation behavior of alloy components near the bulk stoichiometric composition. It is caused by the discontinuity in the effective chemical potential at the stoichiometric composition. The effect is predicted to occur at the (111) surface of ordered $\mathrm{Ni}_{3} \mathrm{Al}$ and $\mathrm{Pt}_{3} \mathrm{Fe}$ alloys.
\end{abstract}

DOI: $10.1103 /$ PhysRevB.65.174201

PACS number(s): 68.35.Dv

The surface composition of alloys is generally different from that of the bulk. In a random metallic alloy, for example, the surface composition is mainly determined by the segregation of one of the alloy components to the surface of the alloy according to Gibbs' adsorption isotherm, ${ }^{1}$ which predicts that the element, which lowers the surface energy, segregates toward the surface. In the case of ordered alloys, however, the situation may be quite different (for a review see Ref. 2). For instance, most of the surface truncations of a perfectly ordered alloy have a composition different from that of the bulk simply for crystallographic reasons. In such cases there are at least two different possible truncations of the bulk, and which of these is realized may not depend on the segregation energy at all. Instead, the surface composition may be connected to the way the surface has been created or even to the evaporation rate of the alloy constituents as seen in the cases of some oxides and carbides. ${ }^{3}$

In this paper I consider the surface of a binary, ordered, and near-stoichiometric alloy with two crystallographically nonequivalent sublattices. We show that not only is the composition of such a surface a function of the surface segregation as well as the ordering in the bulk and at the surface, it is also strongly influenced by the alloy composition in the bulk. In fact, I predict a sharp transition in the segregation behavior of the alloy components caused by an infinitesimal change in the bulk composition near the stoichiometric value. To my knowledge, such a transition has not been observed experimentally, but here, I demonstrate the basis of density functional calculations that the effect should be found at the (111) surface of intermetallic compounds $\mathrm{Ni}_{3} \mathrm{Al}$ and $\mathrm{Pt}_{3} \mathrm{Fe}(111)$.

Both $\mathrm{Ni}_{3} \mathrm{Al}$ and $\mathrm{Pt}_{3} \mathrm{Fe}$ have an $A_{3} B-L 1_{2}$ - or $\mathrm{Cu}_{3} \mathrm{Au}$-type structure with two nonequivalent sublattices. The (111) surface of the perfectly ordered alloys, shown in Fig. 1, has exactly the bulk stoichiometric composition. The equilibrium surface composition at zero temperature (the role of the temperature will be discussed in another paper $\left.{ }^{4}\right)$ is determined by the minimum in the surface energy, $\gamma\left(\left\{c_{i}\right\}\right)$, which depends on the concentration profile $\left\{c_{i}\right\}$ of some finite surface region:

$$
\gamma\left(\left\{c_{i}\right\}\right)=E_{\text {tot }}^{\text {surf }}\left(\left\{c_{i}\right\}\right)-N E_{\text {tot }}^{\text {bulk }}-\mu \sum_{i=1}^{N}\left(c_{i}-c\right),
$$

where $E_{\text {tot }}^{\text {surf }}\left(\left\{c_{i}\right\}\right)$ is the total energy of the surface region per surface atom, $E_{\text {tot }}^{\text {bulk }}$ the total energy of the bulk per atom, $N$ the number of layers in the surface region, $c \equiv c_{B}$ the concentration of the $B$ component in the bulk, and $\mu$ the effective chemical potential. The latter is equal to the difference in the chemical potentials of the two alloy components in the bulk and is given by

$$
\mu=\frac{\partial E(c)}{\partial c}
$$

Here, the concentration derivative should be taken along the minimal path in the space of short- and long-range order (LRO) parameters, where the total energy (per atom) of the alloy, $E(c)$, reaches its minimal value for every value of $c$. Strictly speaking, there could be a phase separation at $T=0$ if $c$ is different from the corresponding stoichiometric value.

$$
\mathrm{L}_{2}(111)
$$

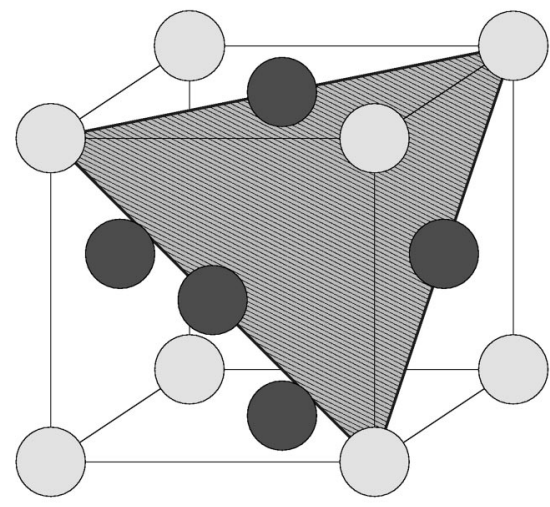

Top view

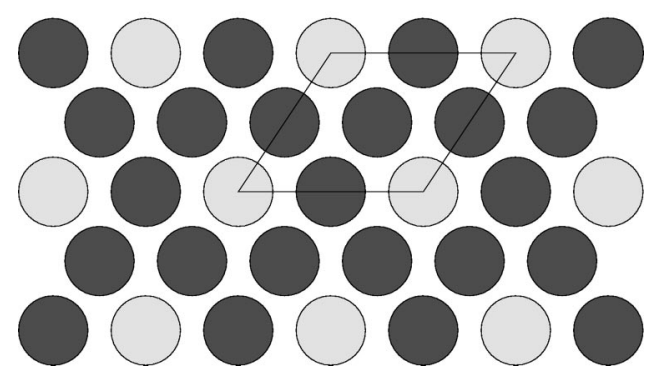

FIG. 1. The structure of the stoichiometric $L 1_{2}(111)$ surface. 


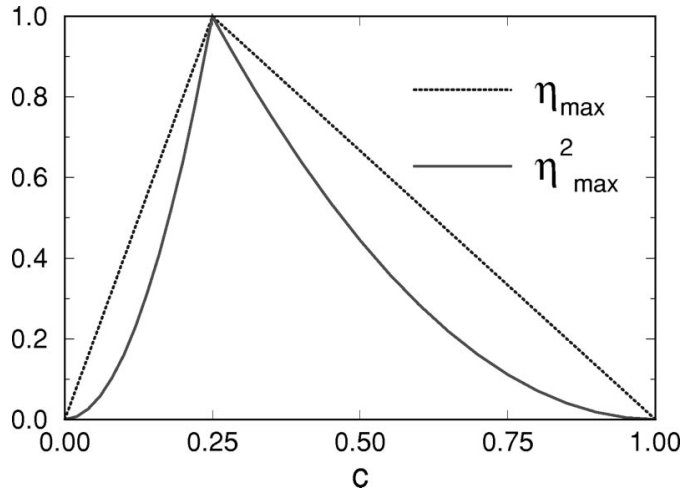

FIG. 2. The maximal value of the LRO parameter as a function of concentration.

However, we will follow a single-phase consideration, since both $\mathrm{Ni}_{3} \mathrm{Al}$ and $\mathrm{Pt}_{3} \mathrm{Fe}$ exist in a final concentration interval.

At $T=0$ the minimal path is trivially identified in $\mathrm{Ni}_{3} \mathrm{Al}$ and $\mathrm{Pt}_{3} \mathrm{Fe}$ since the vacancies do not form stable alloys on the sublattices at off-stoichiometric compositions. Therefore the excess of atoms in the alloy leads only to the formation of the corresponding partial antisite defects, e.g., Ni partial antisites on the Al sublattice in the Ni-rich off-stoichiometric $\mathrm{Ni}_{3} \mathrm{Al}$ alloys. Further, in the vicinity of the stoichiometric composition the role of short-range-order effects due to, for instance, the additional ordering of antisite defects on a sublattice is again negligible, ${ }^{5}$ and, thus, the total energy is basically only a function of the alloy concentration and a LRO parameter, which may be determined as $\eta=c_{B}(B)-c_{B}(A)$, where $c_{B}(\alpha)$ is the concentration of $B$ on the $\alpha$ sublattice.

Since there are no thermal defects at $T=0$, which in the case of $\mathrm{Ni}_{3} \mathrm{Al}$ and $\mathrm{Pt}_{3} \mathrm{Fe}$ are exchange antisite defects or coupled pairs of partial antisites preserving the alloy composition, ${ }^{6,7}$ the maximum value $\eta_{\text {max }}$ providing the lowest ordering and total energy is uniquely determined by the alloy composition $c$ (Ref. 6):

$$
\eta_{\text {max }}= \begin{cases}4 / 3(1-c) & \text { if } c>0.25 \\ 4 c & \text { if } c<0.25\end{cases}
$$

The easiest way to follow the behavior of the total energy of an ordered alloy near the stoichiometric composition is to divide it into two contributions: (i) the total energy of the random alloy of a given composition and (ii) the ordering energy. To lowest order the latter is a quadratic function of the LRO parameter with a prefactor which is the Fourier transform of the effective pair interactions at the corresponding superstructure vector $V\left(\mathbf{k}_{s}\right)$ (Ref. 6):

$$
E_{\text {ord }}=\frac{3}{32} V\left(\mathbf{k}_{s}\right) \eta^{2}(c)
$$

According to Eq. (3) the LRO parameter as a function of alloy composition reaches its maximum value at the stoichiometric composition $c=0.25$ and decreases linearly to both sides. This means that the ordering energy will exhibit a kink as shown in Fig. 2. Since the total energy of a random alloy is usually a smooth function of the alloy composition, the

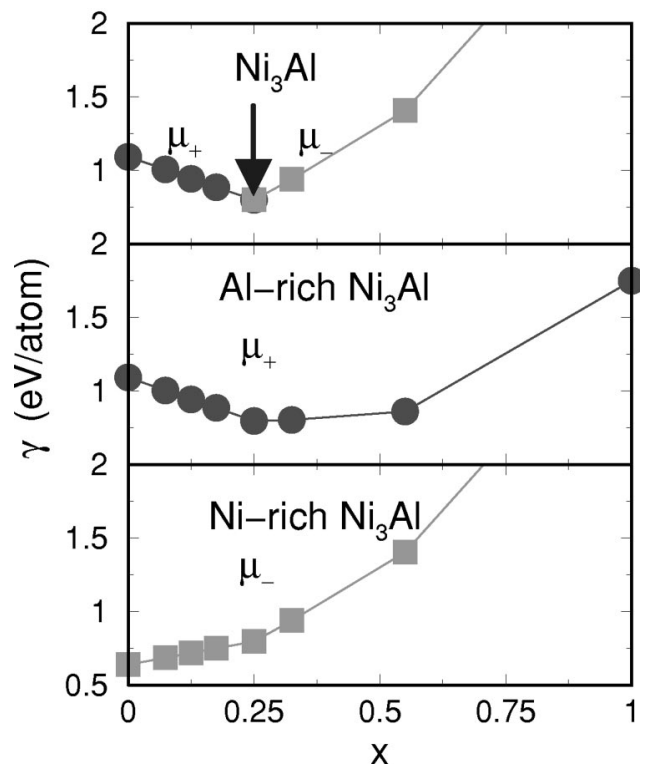

FIG. 3. Surface energy of $\mathrm{Ni}_{1-x} \mathrm{Al}_{x} / \mathrm{Ni}_{3} \mathrm{Al}$ (top panel), $\mathrm{Ni}_{1-x} \mathrm{Al}_{x} / \mathrm{Ni}_{3}\left(\mathrm{Al}_{1-\delta} \mathrm{Ni}_{\delta}\right)$ (middle panel), and $\left(\mathrm{Ni}_{1-\delta} \mathrm{Al} \delta\right)_{3} \mathrm{Al}$ (lower panel). $\delta \rightarrow 0$.

kink will be present in the total energy of the ordered alloy and, consequently, the chemical potential will exhibit a discontinuity at the stoichiometric composition. Using the definition of the effective chemical potential (2) and the ordering energy (4) together with Eqs. (3) and (5) one finds that the discontinuity is exactly equal the energy of the exchange antisite defect, $\varepsilon_{x c}$, which in the case of the $L 1_{2}$ structure is ${ }^{6}$

$$
\varepsilon_{x c}=\frac{16}{3} \frac{\partial E_{o r d}}{\partial \eta}=V\left(\mathbf{k}_{s}\right) \eta .
$$

Thus, if the chemical potential for $A$-rich alloys ( $c$ $<0.25)$ is $\mu_{-}$and the chemical potential for the $B$-rich alloys $(c>0.25)$ is $\mu_{+}$, then $\mu_{+}=\mu_{-}+\varepsilon_{x c}$. At the stoichiometric composition there are two different chemical potentials $\mu_{+}$and $\mu_{-}$. However, only one of these enters the definition of the surface energy (1) depending on the sign of $\Delta c=\Sigma_{i}\left(c_{i}-c_{b}\right)$ : If $\Delta c>0$, i.e., $B$ atoms segregate toward the surface region whereby the bulk composition becomes $A$ rich, $\mu=\mu_{-}$, and if $\Delta c<0$, i.e., $A$ atoms segregate toward the surface region and the bulk is enriched by the $B$ component, $\mu=\mu_{+}$.

In Fig. 3 we show the calculated surfaces energies of $\mathrm{Ni}_{1-x} \mathrm{Al}_{x}(111) / \mathrm{Ni}_{3} \mathrm{Al}$ as functions of the $\mathrm{Al}$ concentration $(x)$ in the surface layer for the stoichiometric and for two off-stoichiometric, Al-rich and Ni-rich, bulk compositions. In all the calculations the alloy in the surface layer has been considered to be in the maximally ordered $L 1_{2}(111)$ state, i.e., without exchange antisite defects. The partial antisites of one type have been assumed to be distributed randomly on the sublattice of the deficient element. The calculations have been performed by the Green's function technique based on the Korringa-Kohn-Rostoker (KKR) method in the atomic sphere approximation with multiple moment correction for 


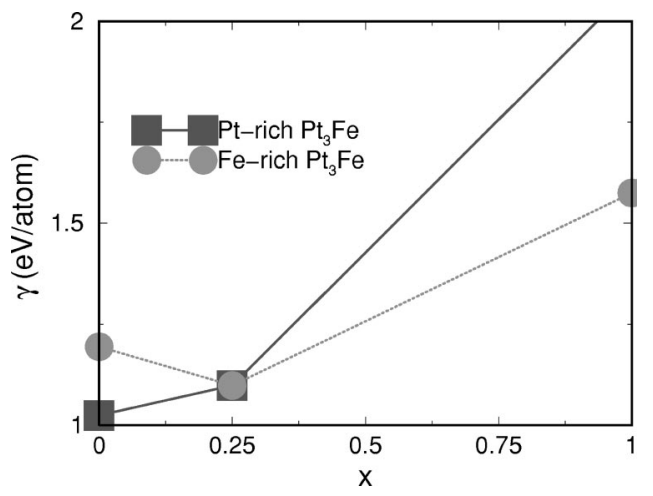

FIG. 4. Surface energy of $\mathrm{Pt}_{1-x} \mathrm{Fe}_{x} / \mathrm{Pt}_{3} \mathrm{Fe}$ for Pt- and Fe-rich alloy compositions. The surface layer is in the maximally ordered state.

the Madelung potential and energy. ${ }^{8}$ The coherent potential approximation has been used to obtain the electronic structure of the partially ordered alloys in the bulk (during the chemical potential calculations) and at the surface. The local density approximation has been used with the exchangecorrelation functional parametrized by Perdew et al. ${ }^{9}$ More details about the Green's function technique may be found in Ref. 8.

Lattice relaxation effects due to the atomic size mismatch of the alloy components, which may be important for an accurate quantitative description of the alloy energetics in these systems, have not been included. Since the (111) facet is closed packed, this, in fact, is not important for the segregation energies since the corresponding contributions to the bulk and surface chemical potentials are usually cancelled out. ${ }^{10}$ The largest error due to the interlayer relaxations will be in those cases where the alloy composition at the surface is significantly different from that of the bulk, that is, when the surface of an alloy is covered by a psuedomorphic monolyaer of one of the alloy components. In the case of $\mathrm{Ni}$ on $\mathrm{Ni}_{3} \mathrm{Al}(111)$ and $\mathrm{Pt}$ on $\mathrm{Pt}_{3} \mathrm{Fe}(111)$ the relaxations, however, should be small due to the small lattice mismatch of bulk $\mathrm{Ni}$ $(\mathrm{Pt})$ and $\mathrm{Ni}_{3} \mathrm{Al}\left(\mathrm{Pt}_{3} \mathrm{Fe}\right)$. In the case of $\mathrm{Al}$ on $\mathrm{Ni}_{3} \mathrm{Al}(111)$ and $\mathrm{Fe}$ on $\mathrm{Pt}_{3} \mathrm{Fe}(111)$ the interlayer relaxations could be of order $0.1 \mathrm{eV}$, since the corresponding interlayer relaxations for $\mathrm{Al}$ on $\mathrm{Ni}(111)$ and $\mathrm{Fe}$ on $\mathrm{Pt}(111)$ are about 0.04 and $0.16 \mathrm{eV}$, respectively. ${ }^{11}$ However, such relaxations may change only the slope of the corresponding surface enery curves near the corresponding surface configurations. This hardly influences the quilitative consideration adopted in this paper.

Consider now the surface energy for the offstoichiometric alloy compositions shown in Fig. 3. In the $A$-rich alloy the bulk alloy composition is $A_{3}\left(B_{1-\delta} A_{\delta}\right)$ while in $B$-rich alloy $\left(A_{1-\delta} B_{\delta}\right)_{3} B$, where $0<\delta<1$. In fact, the results presented in the figure are obtained for $\delta \rightarrow 0$; i.e., the actual difference in the bulk compositions of the two off-stoichiometric alloys is negligible. Nonetheless, the surface energies differ dramatically in the two cases and, as a consequence, the surface layer in the Al-rich alloy should be $\mathrm{Ni}_{3} \mathrm{Al}(111)$, while, according to the lower panel, Ni should segregate toward the surface of the $\mathrm{Ni}$-rich $\mathrm{Ni}_{3} \mathrm{Al}$, forming a pure $\mathrm{Ni}$ overlayer. Finally, if the bulk composition is exactly stoichiometric, the surface composition is pinned at the stoichiometric value. In other words, the $\mathrm{Ni}$ concentration in the surface layer changes discontinuously from 0.75 to 1.00 upon an infinitesimal change in the bulk concentration.

The kink in the surface energy at the stoichiometric composition of the surface alloy $(x=0.25)$ in an offstoichiometric bulk alloy is the consequence of the behavior of the ordering energy in the surface layer, which is similar to that of the bulk discussed earlier. The kink, however, is almost doubled in magnitude at the exact stoichiometric bulk composition, because of the discontinuous behavior of the effective chemical potential. This means that the segregation energy of, e.g., $\mathrm{Al}$, toward the surface layer, obtained as the first derivative of the surface energy with respect to the concentration $(x)$ in the first layer, has a discontinuity at the stoichiometric composition for the surface alloy $(x=0.25)$ of $\varepsilon_{x c}^{\text {surf }}+\varepsilon_{x c}$, where $\varepsilon_{x c}^{\text {surf }}$ is the energy of the formation of an exchange antisite defect in the ordered surface layer. In the case of $\mathrm{Ni}_{3} \mathrm{Al}, \varepsilon_{x c}=0.98 \mathrm{eV}$ and $\varepsilon_{x c}^{\text {surf }}=1.0 \mathrm{eV}$. One might have expected a reduction in the latter compared to the bulk value due to the reduced number of bonds at the surface, but it is found to be almost unchanged due to the enhanced ordering at the surface, which seems to be a common effect for the $\mathrm{NiAl}$ system. ${ }^{8}$ Hence, the segregation energy is changed by about $2 \mathrm{eV}$ by an infinitesimal change in the surface alloy composition near its stoichiometric value.

The physical mechanism behind the unusual behavior of the surface and segregation energies is as follows. Consider the difference in the surface energy of Al-rich and Ni-rich bulk $\mathrm{Ni}_{3} \mathrm{Al}$ for a surface alloy composition in the range 0 $<x<0.25$. Such a composition corresponds to the segregation of Ni toward the surface layer which involves the transfer of Ni atoms from the bulk to the surface and a compensating transfer of $\mathrm{Al}$ atoms from the surface into the bulk. If the bulk alloy composition is $\mathrm{Al}$ rich, this can happen only by the formation of additional, partial $\mathrm{Al}$ antisite defects on the Ni sublattice, which is always energetically unfavorable. Hence, the surface energy increases. If, on the other hand, the bulk alloy is $\mathrm{Ni}$ rich, the $\mathrm{Al}$ atoms that arrive from the surface go to the $\mathrm{Al}$ sublattice where they replace $\mathrm{Ni}$ atoms which go to the surface, thereby annihilating partial $\mathrm{Ni}$ antisite defects on the Al sublattice in the bulk. This is an energetically favorable process and the surface energy decreases accordingly.

The reason why the surface energy of stoichiometric $\mathrm{Ni}_{3} \mathrm{Al}$ viewed as function of surface composition $x$ is a combination of the two upper branches of the surface energies for the Al-rich and Ni-rich cases (see Fig. 3) is the fact that any change in the surface composition relative to the stoichiometric value leads to the formation of partial antisites in the bulk. As a result, the surface composition becomes pinned to the stoichiometric value. This is in agreement with existent experimental data ${ }^{12,13}$ which indicate that the (111) surface of stoichiometric $\mathrm{Ni}_{3} \mathrm{Al}$ alloy has the stoichiometric composition.

To observe the discontinuous change in the surface composition experimentally one must produce a slightly Ni-rich $\mathrm{Ni}_{3} \mathrm{Al}$ sample. However, at very small deviations from the stoichiometric bulk composition the concentration of antisite 
defects will be very small and most of the antisite defects will be very far from the surface. Hence, the formation of the equilibrium concentration profile will be kinetically hindered, especially at low temperatures. On the other hand, at high temperatures the off-stoichiometric effect will be less pronounced, since the formation energy for antisite defects, which depend linearly on the LRO parameters (5), will be smaller.

In Fig. 4, I show the similar off-stoichiometric effect at the (111) surface of ordered $\mathrm{Pt}_{3} \mathrm{Fe}$. Here, the surface of the Pt-rich alloy is completely covered by $\mathrm{Pt}$ while the surface composition of the Fe-rich alloy should be stoichiometric, at least at temperatures well below the order-disorder transition temperature of $1400 \mathrm{~K}$. These results are partially confirmed by several experimental investigations ${ }^{14,15}$ which have established that the (111) surface of ordered $\mathrm{Pt}_{80} \mathrm{Fe}_{20}$ is almost entirely covered by Pt atoms. Unfortunately, no other compositions of this ordered alloy have been investigated.
${ }^{1}$ J. W. Gibbs, Collected Works (Yale University Press, New Haven, CT, 1945), Vol. 1.

${ }^{2}$ M. Polak and L. Rubinovich, Surf. Sci. Rep. 38, 127 (2000).

${ }^{3}$ K. E. Tan, M. W. Finnis, A. P. Horsefield, and A. P. Sutton, Surf. Sci. 348, 49 (1996).

${ }^{4}$ L. V. Pourovskii, A. V. Ruban, B. Johansson, and I. A. Abrikosov (unpublished).

${ }^{5}$ M. A. Krivoglaz, Diffuse Scattering of X-Rays and Neutrons by Fluctuations (Springer, Berlin, 1996).

${ }^{6}$ A. V. Ruban and H. L. Skriver, Phys. Rev. B 55, 856 (1997).

${ }^{7}$ P. A. Korzhavyi, I. A. Abrikosov, B. Johansson, A. V. Ruban, and H. L. Skriver, Phys. Rev. B 59, 11693 (1999).

${ }^{8}$ A. V. Ruban and H. L. Skriver, Comput. Mater. Sci. 15, 119 (1999).

${ }^{9}$ J. P. Perdew, K. Burke, and M. Ernzerhof, Phys. Rev. Lett. 77, 3865 (1996).
${ }^{10}$ A. Christensen, A. V. Ruban, P. Stoltze, K. W. Jacobsen, H. L. Skriver, and J. K. Nørskov, Phys. Rev. B 56, 5822 (1997).

${ }^{11}$ These results have been obtained in the density functional theory (generalized gradient approximation) ultrasoft norm-conserving pseudopotential calculations for a six-layer slab in which the positions of the first two layers were allowed to relax.

${ }^{12}$ D. Sondericker, F. Jona, and P. M. Marcus, Phys. Rev. B 34, 6770 (1986).

${ }^{13}$ C. Becker, J. Kandler, H. Raaf, R. Linke, T. Pelster, M. Drager, M. Tanemura, and K. Wandelt, J. Vac. Sci. Technol. A 16, 1000 (1998).

${ }^{14}$ P. Becaat, Y. Gauthier, R. Baudoing-Savois, and J. C. Bertolini, Surf. Sci. 238, 105 (1990).

${ }^{15}$ C. Creemers and P. Deurinck, Surf. Interface Anal. 25, 177 (1997). 